\title{
Mobile Tablet-based Stroke Rehabilitation: Using mHealth Technology to Improve Access to Early Stroke Rehabilitation
}

https://doi.org/10.3991/ijim.v11i1.6234

M.W. Pugliese

The University of Ottawa, Ottawa, Canada

mpugl038@uottawa.ca

K. Wilson

The University of Ottawa, Ottawa, Canada

Ottawa Hospital Research Institute, Ottawa, Canada

kwilson@ohri.ca

J. Guerinet

Ottawa Hospital Research Institute, Ottawa, Canada jguerineteohri.ca

K.M. Atkinson

Ottawa Hospital Research Institute, Ottawa, Canada

Karolinska Institute, Stockholm, Sweden

katkinson@ohri.ca

K.H. Mallet

Ottawa Hospital Research Institute, Ottawa, Canada The Ottawa Hospital, Ottawa, Canada

kmalletetoh.ca

R. Shamloul

Ottawa Hospital Research Institute, Ottawa, Canada rshamlouleohri.ca

\section{Zakutney}

Ottawa Hospital Research Institute, Ottawa, Canada The Ottawa Hospital, Ottawa, Canada

lzakutney@toh.on.ca

D. Corbett

Canadian Partnership for Stroke Recovery, Ottawa, Canada University of Ottawa Brain and Mind Institute, Ottawa, Canada dcorbett@uottawa.ca

D. Dowlatshahi

Ottawa Hospital Research Institute, Ottawa, Canada University of Ottawa Brain and Mind Institute, Ottawa, Canada ddowlatetoh.ca 


\begin{abstract}
Mobile health (mHealth) technology represents a means through which more stroke survivors could access early stroke rehabilitation. Although rehabilitation is most effective when begun early post-stroke, limited resources (facilities, therapists) prevent survivors from initiating therapy. Furthermore, the coupling of an aging population with advances in acute therapy has led to an increase in the absolute number of individuals suffering from and surviving strokes which in turn has put further strain on already scarce rehabilitation resources. There is an urgency to conduct high-quality research exploring costeffective and creative mHealth devices for early rehabilitation in the acute setting. Mobile technology allows therapists to prescribe apps based on standard cognitive/physical assessments in the acute setting, remotely monitor patient progress across individual carepaths, and update prescribed therapies based on patient feedback and recovery. Recognition of the growing problem of accessing early stroke rehabilitation, and the possibilities offered by mHealth technology led to the development of the RecoverNow platform for stroke rehabilitation in the acute setting. RecoverNow is a custom built, tablet-based stroke rehabilitation platform that houses a variety of previously existing apps with activities analogous or identical to exercises in speech language and/or occupational therapy. While RecoverNow represents how mobile technology can be utilized to address a growing public health issue, the feasibility, acceptability and efficacy of tablet-based stroke rehabilitation are unknown. Studies with the goal of establishing feasibility of early tablet-based stroke rehabilitation are needed and, if appropriate, a randomized controlled trial to establish efficacy.
\end{abstract}

Keywords - aphasia, mobile tablet, recovery, stroke.

In the years to come, the stroke community will be tasked with solving an increasingly important problem: how can early stroke rehabilitation be made accessible to a continuously growing population of stroke survivors when resources are already unable to cope with current needs? Globally in 2010, 16.9 million individuals suffered a first ever stroke and there were 33 million stroke survivors representing increases of $68 \%$ and $84 \%$ respectively since 1990 [1]. Among the growing number of stroke survivors, one-third of survivors experience aphasia [2], up to two-thirds experience cognitive impairments [3], and another third experience post-stroke depression [4]. These life-altering impairments can prevent individuals from returning to work, engaging in preferred regular activities, and in some cases, prevent independent living.

When combined with acute stroke therapy, stroke rehabilitation reduces odds of death, dependency, and institutionalization [5]. Although the optimal time for beginning stroke rehabilitation has not been identified [6], there is considerable evidence showing that better results are obtained when rehabilitation begins early post-stroke and when therapies are performed intensely [7]. However, many patients are not receiving timely rehabilitation. In Canada, the proportion of patients transferred to inpatient rehabilitation is approximately $16 \%$, even though $40 \%$ of patients would benefit from inpatient rehabilitation [8]. Among those transferred, only 50\% begin rehabilitation within two weeks. These patients spend an average of eight extra days in acute 
care between the completion of acute therapy and assessments, and transfer to rehabilitation facilities [9], often due to resource limitations and shortages in rehabilitation spaces [10]. A similar pattern is seen in the United States with $24 \%$ of patients being transferred to inpatient rehabilitation after an average of 27 days from stroke onset [11][12]. Patients experience significant down-time during their acute care stay, spending more than $50 \%$ of their time resting in bed and only $13 \%$ of time engaging in recovery [13]. It is during this down-time where the proposed mHealth strategy to improve access to early stroke rehabilitation could be implemented.

\section{The mHealth Strategy}

Mobile health technology may be able to provide a solution to increase the availability of early stroke rehabilitation through relatively economically-efficient means. Mobile technology has become ubiquitous and affordable, and if carefully developed in collaboration with health experts and evaluated using robust scientific methodology, this technology can be used to effectively provide therapy. In particular, tablet computers are of interest as they are compact yet large enough to be easily manipulated by stroke survivors. Recently, there have been a number of studies exploring the feasibility and effectiveness of various tablet-based stroke therapies and stakeholder perspectives on their usage [14]-[18]. Although these studies are typically small pilot studies of varying quality, they reflect the growing interest in tablets as a means of providing therapy.

Transforming a tablet into a stroke rehabilitation platform would reduce patient dependence on bedside therapist visits for engagement in rehabilitation activities while in acute care. Instead, a streamlined approach can be taken where a therapist assesses a patient and prescribes a personalized rehabilitation regime directly through the platform. Between therapist assessments, medical tests and other acute care activities, patients could independently engage in their prescribed rehabilitation activities. Their progress could be monitored remotely, and regimes adjusted to reflect their current state - all without a bedside visit. Furthermore, patients would have access to rehabilitation regardless of how they proceeded through the continuum of care; whether they continue to inpatient rehabilitation or directly to the community, as the device is easily portable. How to go about harnessing the power of mHealth technology to provide tablet-based stroke rehabilitation is dependent on close collaboration between software developers and stroke rehabilitation experts.

\section{RecoverNow: A Mobile Tablet-Based Platform for Early Stroke Rehabilitation}

The need for a cost-efficient means of improving access to early rehabilitation paired with the power and affordability of current mobile technology led to the development of RecoverNow at The Ottawa Hospital. RecoverNow is an Android tabletbased early stroke rehabilitation platform designed for use in the acute care setting. 
The platform is a custom Android launcher (home screen) limiting the patient to a list of tailored therapeutic apps that can be swapped in and out by therapists, depending on patient rehabilitation needs (Figure 1). By harnessing tablet technology and currently available apps, patients can begin speech language therapy and occupational therapy while in acute care, and use the device to supplement therapy during inpatient or outpatient rehabilitation. RecoverNow empowers stroke survivors with the ability to control when and how often they choose to engage in rehabilitation, while still receiving guidance from rehabilitation professionals.

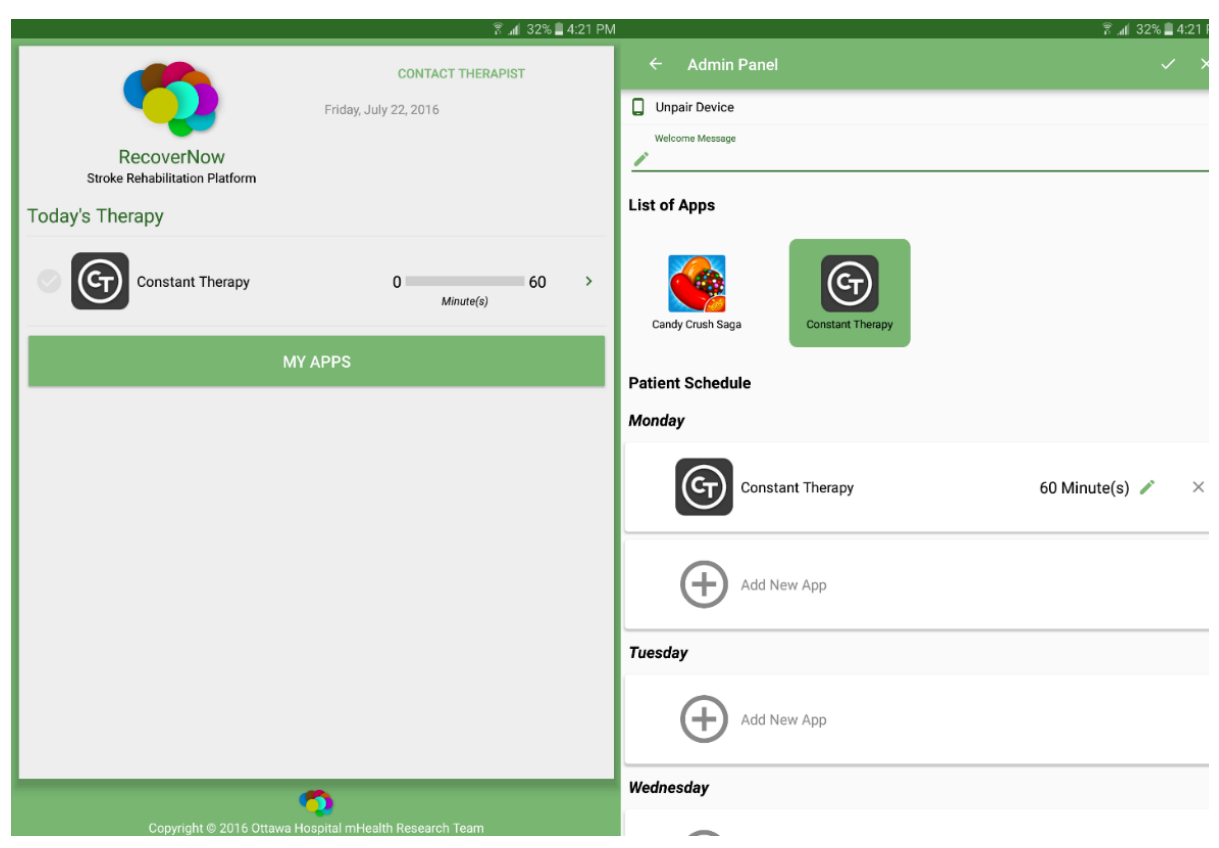

Fig. 1. Tablet screenshot of RecoverNow patient homepage (left) and therapist administration portal (right)

Once patients are admitted to an acute care facility for stroke and have completed the standard of care allied health assessments, early rehabilitation can begin via mobile tablet. Based on the assessments, patients and their therapists can select apps best suited towards their specific deficits and recovery goals. The included apps are selected from pre-existing products in the Google Play Store, that may either be directly therapy-related (ex: Constant Therapy for aphasia and cognition) or simply games that are selected by therapists to help improve certain motor, cognitive, linguistic and/or perceptual processes (ex: Fruit Ninja for cuing, fine motor control and frontal inhibition). Furthermore, a Patient Health Questionnaire (PHQ-9) app allows for quick and easy depression screening. As soon as apps have been selected and app usage goals assigned, the patient is ready to begin tablet-based therapy.

While engaging in therapy between regularly scheduled tests and assessments, patients can use the cellular capabilities of the tablet to keep in contact with their thera- 
pist. A messaging system using a pre-defined list of messages can be used to communicate technical difficulties or displeasure with the recommended apps. A desktop administration portal allows therapists to remotely update the apps patients can access on their tablets based on patient feedback or follow-up assessments. The administration portal can also be used to change recommended app usage goals and track the amount of time patients have spent engaging in therapy. Based on app usage data, therapists can identify patients who have difficulty adhering to the prescribed therapeutic regimen, send personal messages of encouragement, or prescribed less challenging apps (Figure 2). All of this information can be transmitted and received in real-time via a cellular connection, thereby avoiding the need to use institutional wireless networks thus alleviating some security concerns.

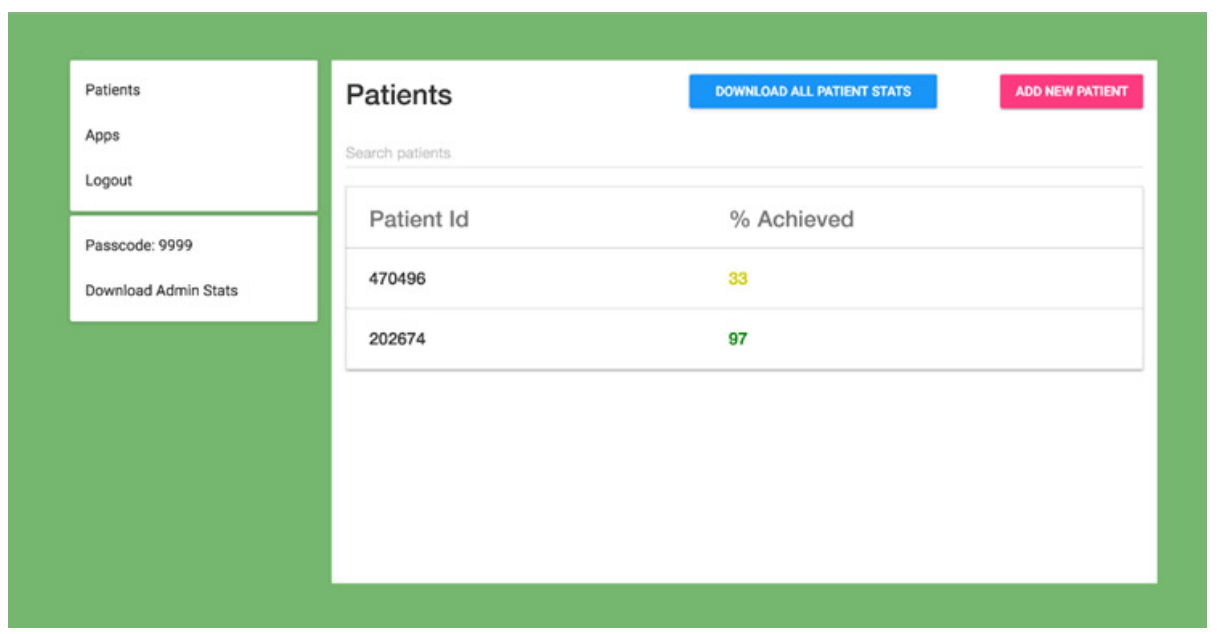

Fig. 2. RecoverNow web administration portal. Patients with app usage achievement below $50 \%$ of recommended time are highlighted for easy identification

Therapist-patient communication and regular assessments create a cyclical process where recommended apps continually change based on patient feedback, adherence and recovery progress. This cyclical prescribe-engage-assess process can continue remotely as patients continue to recover (Figure 3). Rehabilitation with the remote guidance of a therapist can similarly continue after discharge, and throughout the transitions of care.

\section{$3 \quad$ Limitation of Tablet-Based Stroke Rehabilitation}

Tablet-based stroke rehabilitation platforms like RecoverNow are an economically attractive means of offering early rehabilitation to patients who would otherwise receive little to no immediate therapy while in acute care. However, this solution is limited in that it currently lacks the full comprehensiveness of a typical stroke rehabilitation program. In particular, physiotherapy is difficult to provide without supervi- 
Short Paper-Mobile Tablet-based Stroke Rehabilitation: Using mHealth Technology to Improve Access to Early Stroke Rehabilitation

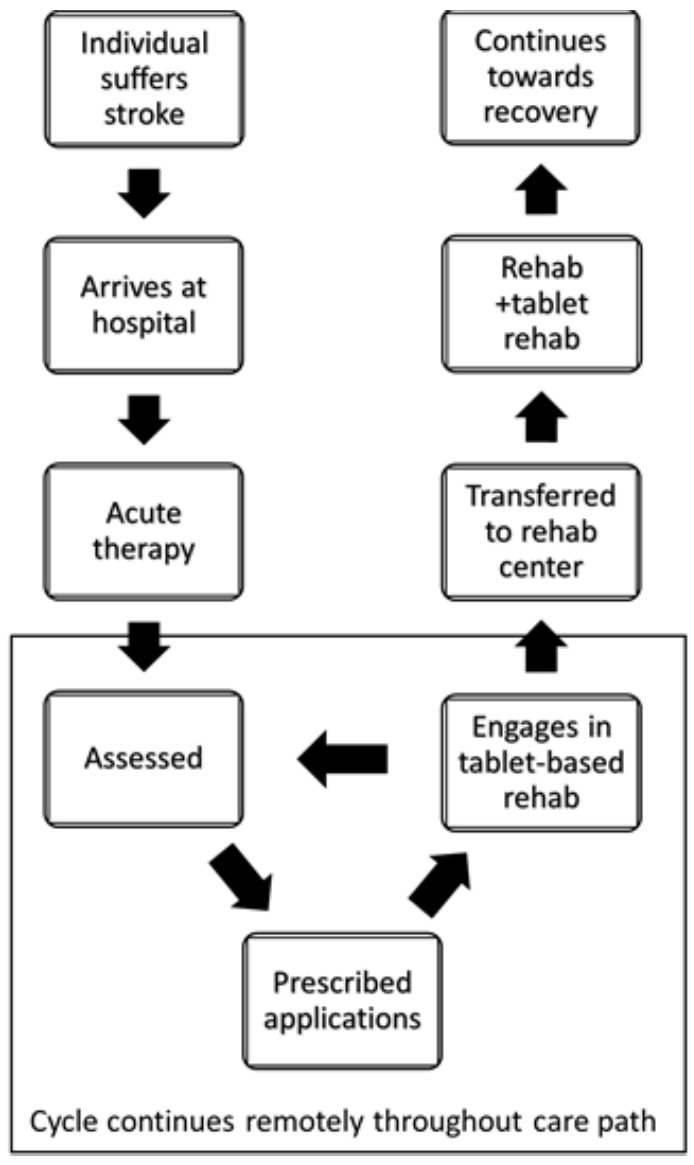

Fig. 3. Use-case scenario of tablet-based rehabilitation in the stroke-care pathway

sion to ensure safety in the early post-stroke setting. There are also limits to which stroke survivors will be able to manipulate tablets in order to participate in therapy. Individuals without a functional upper limb may have limitations using tablet-based therapies, as would individuals with severe comprehension deficits. It is therefore unclear if tablet-based therapies are feasible for patients with more severe strokes.

Even if feasible for patients, the acceptability of tablet-based therapy is unclear. Some patients may have concerns about their anonymity using platforms that capture and transmit detailed participant usage data including apps accessed, the length of the app usage session, and the date and time of each app usage session. Online platforms also carry the risk of security and privacy breaches that may be concerning to some patients due to the collection and storage of the potentially sensitive personal usage data listed above. However, little is known about tablet-based therapy and the communication of this information is crucial as it is needed to evaluate patient adherence with the daily app usage recommended by therapists, and therefore the feasibility of offering tablet-based stroke rehabilitation in the acute setting. 


\section{$4 \quad$ Next Steps}

There is an urgency to pursue the rigorous development and evaluation of tabletbased strategies for increasing accessibility to early stroke rehabilitation. Both the health system need and mobile technology are present, what remains is to answer questions regarding feasibility, acceptability and efficacy. Although there are some small pilot studies supporting the feasibility of tablet-based therapy for stroke patients [14][16], there appears to be no evidence regarding acute patients. Mobile devices and rehabilitation platforms/apps need to be put into the hands of acute stroke patients in the form of well-designed longitudinal studies in order to establish therapy adherence, barriers to use, and to generate patient feedback that can be integrated into the design of tablet-based therapy platforms and apps. Once deemed feasible and acceptable, a randomized controlled trial would be appropriate to determine if tablet-based therapy in addition to standard stroke rehabilitation results in better functional outcomes when compared to standard care alone.

\section{Conclusion}

There are currently not enough resources to meet the demand for early stroke rehabilitation and without cost-effective solutions the demand is likely to continue rising above the limited number of available therapists and inpatient rehabilitation beds. It follows that these barriers to early therapy will prevent patients from achieving maximum recovery and increase the number of survivors requiring some form of assistedliving. This public health problem could potentially be addressed using mobile technology, specifically mobile tablet-based stroke rehabilitation. Tablet-based rehabilitation has the potential to empower stroke survivors to take an early active role towards recovery. This promising strategy requires evaluation to establish feasibility and acceptability before attempting to establish efficacy. If successful, more stroke survivors will be able to maximize their recovery potential through easy access to early stroke rehabilitation.

\section{References}

1. V.L. Feigin et al., "Global and regional burden of stroke during 1990-2010: findings from the Global Burden of Disease Study 2010," Lancet, vol. 383, pp. 245-255, Oct. 2014. https://doi.org/10.1016/S0140-6736(13)61953-4

2. K. Salter, R. Teasell, N. Foley, and L. Allen. (2013, September). Evidence-based systematic review of stroke rehabilitation: Aphasia $\left(16^{\text {th }}\right.$ ed. $)[$ Online]. Available: http://www.ebrsr.com/evidence-review/14-aphasia.

3. K. Salter, R. Teasell, N. Foley, and L. Allen. (2013, July). Evidence-based systematic review of stroke rehabilitation: Cognitive disorders and apraxia $\left(16^{\text {th }}\right.$ ed.)[Online]. Available: http://www.ebrsr.com/evidence-review/12-cognitive-disorders-and-apraxia. 
4. K. Salter et al., (2013, August). Evidence-based systematic review of stroke rehabilitation: Post stroke depression (16 $6^{\text {th }}$ ed.)[Online]. Available: http://www.ebrsr.com/evidencereview/18-post-stroke-depression.

5. N. Foley, R. Teasell, S. Bhogal, M. Speechley, and N. Hussein. (2013, November). Evidence-based review of stroke rehabilitation: The efficacy of stroke rehabilitation $\left(16^{\text {th }}\right.$ ed.)[Online]. Available: http://www.ebrsr.com/evidence-review/5-efficacy-strokerehabilitation.

6. J. Bernhardt et al., "Efficacy and safety of very early mobilization within $24 \mathrm{~h}$ of stroke onset (AVERT): a randomised controlled trial," Lancet, vol. 386, pp. 46-55, Apr. 2015. https://doi.org/10.1016/S0140-6736(15)60690-0

7. R. Teasell and N. Hussein. (2013, November). Evidence-based review of stroke rehabilitation: Background concepts in stroke rehabilitation (16 th ed.)[Online]. Available: http://www.ebrsr.com/evidence-review/3-background-concepts-stroke-rehabilitation.

8. Heart and Stroke Foundation. (2014, June). Stroke report 2014: Together against a rising tide [Online]. Available: http://www.heartandstroke.com/atf/cf/\%7B99452D8B-E7F14BD6-A57D-B136CE6C95BF\%7D/HSF SMReport2014E Final.pdf.

9. Canadian Institute for Health Information. (2012, July). Pathways of care for people with stroke in Ontario July 2012 [Online]. Available: https://secure.cihi.ca/free products/ Pathways of care aib en.pdf.

10. Heart and Stroke Foundation. (2007, April 30). Consensus panel on the stroke rehabilitation system April 2007 [Online]. Available: http://www.heartandstroke.on.ca/atf/ cf/\%7B33C6FA68-B56B-4760-ABC6-D85B2D02EE71\%7D/SRSCP FULL REPORT F INAL20070430\%5B1\%5D.pdf.

11. M. B. Buntin, C. H. Colla, P. Deb, N. Sood, and J. J. Escarce, "Medicare spending and outcomes after postacute care for stroke and hip fractures," Med Care, vol. 48, pp. 776784, Sept. 2010. https://doi.org/10.1097/MLR.0b013e3181e359df

12. H. Wang, M. Camaicia, J. Terdiman, Y. Hung, and M. E. Sandel, "Time to inpatient rehabilitation hospital admission and functional outcomes of stroke patients," Am. J. Phys. Med. Rehabil., vol. 3, pp. 296-304, Apr. 2011. https://doi.org/10.1016/j.pmrj.2010.12.018

13. J. Bernhardt, H. Dewey, A. Thrift, and G. Donnan, "Inactive and alone: physical activity within the first 14 days of acute stroke unit care," Stroke, vol. 35, pp. 1005-1009, July 2004. https://doi.org/10.1161/01.STR.0000120727.40792.40

14. C. Des Roches, I. Balachandran, E. M. Asceno, Y. Tripodies, and S. Kiran, "Effectiveness of impairment-based individualized rehabilitation program using an iPad-based software platform," Front. Neurosci, vol. 8, Article 1015, pp. 1-29, Jan. 2015. https://doi.org/10.3389/fnhum.2014.01015

15. E. L. Hoover and A. Carney, "Integrating the iPad into an intensive, comprehensive aphasia program," Semin. Speech Lang., vol. 35, pp. 25-37, 2014. https://doi.org/10.1055/s0033-1362990

16. J. Kurland, A. R. Wilkins, and P. Stokes, "iPractice: Piloting the effectiveness of a tabletbased home practice program in aphasia treatment," Semin. Speech Lang., vol. 35, pp. 5164, Feb. 2015. https://doi.org/10.1055/s-0033-1362991

17. G. Saposnik et al., "iPad technology for home rehabilitation after stroke (iHOME): A proof-of-concept randomized trial," Int. J. Stroke, vol. 9, pp. 956-962, July 2015. https://doi.org/10.1111/ijs.12328

18. J. White, H. Janssen, L. Jordan, and M. Pollack, "Tablet technology during stroke recovery: a survivor's perspective,” Disabil. Rehabil., vol. 37, pp. 1186-1192, Sept. 2015. https://doi.org/10.3109/09638288.2014.958620 
Short Paper-Mobile Tablet-based Stroke Rehabilitation: Using mHealth Technology to Improve Access to Early Stroke Rehabilitation

\section{$7 \quad$ Authors}

M. W. Pugliese is with the School of Epidemiology, Public Health and Preventive Medicine at The University of Ottawa, Ottawa, Canada. He is a MSc. Epidemiology student studying tablet-based rehabilitation following acute stroke. The Ottawa Hospital, General Campus, 451 Smyth Road, Roger-Guindon Building, Room 3105, Ottawa, ON, K1H8M5.

K. Wilson (corresponding author) is a specialist in General Internal Medicine at the Ottawa Hospital. He is a Professor of Medicine at the University of Ottawa and Senior Scientist in the Clinical Epidemiology Program at the Ottawa Hospital Research Institute. He holds a Research Chair in Public Health Innovation. He has guided a team in the development of mobile health applications within the Ottawa Hospital. The Ottawa Hospital, Civic Campus, 1053 Carling Avenue, Administrative Services Building, Room 1009, Box 684, Ottawa, ON, K1Y4E9 (email: kwilson@ohri.ca).

J. Guerinet is the head of mobile development at the Ottawa Hospital mHealth Research Team. He built the tablet portion of the RecoverNow platform and is currently leading the mobile aspect of multiple other projects, most notably ImmunizeCA. 103B Stirling Avenue, Ottawa, ON, K1Y1P9.

K. M. Atkinson is the Director of Research and Business Development at the Ottawa Hospital mHealth Research Team. She is also a PhD student at the Karolinska Institutet in Stockholm, Sweden where she is studying the use of mobile technologies to improve health outcomes. 103B Stirling Ave, Ottawa, ON, K1Y1P9.

K. H. Mallet has been working as the Speech-Language Pathologist on the Best Practice Team with the Champlain Regional Stroke Network since 2004. Her academic background includes a MEd in Education - Counselling and a MHSc in SpeechLanguage Pathology, both from the University of Ottawa. Karen has lectured at the University of Ottawa's Medical School and Speech-Language Pathology/Audiology Programs. She has been a collaborator in developing the Heart and Stroke's 2010 Tips and Tools for Everyday Living's Meal Assistance and Special Diets Module. She is currently working on a feasibility research project using TeleRehab at Home. The Ottawa Hospital, Box 608, 1053 Carling Avenue, Ottawa, ON, K1Y4E9.

R. Shamloul is a senior clinical research associate at the Ottawa Stroke Program (OSP) at the Ottawa Hospital Research Institute. He has a MBBCH from Cairo University, $\mathrm{PhD}$ from University of Saskatchewan and MBA from Western Governors University. He is involved in the research operational activities of the OSP. The Ottawa Hospital, Box 608, 1053 Carling Avenue, Ottawa, ON, K1Y4E9.

L. Zakutney obtained her Bachelor of Sciences Degree in Occupational Therapy from McGill University. Throughout her career she has worked across Canada in acute care settings, outpatient rehab, and in the private sector. For over 29 years of her career, she has been working with, and dedicated to, the stroke and brain injury population. She has been affiliated with the University of Ottawa since she joined the Champlain Regional Stroke Network in 2007 disseminating Stroke Best Practices in this region in Ontario Canada. The Ottawa Hospital, Civic Campus, 1053 Carling Avenue, Ottawa, ON, K1Y4E9. 
D. Corbett is a neuroscientist and the Scientific Director and CEO of the Heart and Stroke Foundation Canadian Partnership for Stroke Recovery. He obtained a PhD from Concordia University followed by Post-Doctoral training at McGill University. His research deals with questions related to the optimal timing and intensity of poststroke rehabilitation and the biological mechanisms underlying recovery after stroke. The Ottawa Hospital, General Campus, Room 3510G, Roger Guindon Hall, 451 Smyth Rd, Ottawa, ON, K1H8L1.

D. Dowlatshahi is a stroke neurologist and the scientific director of the Ottawa Stroke Program. He has an MD and PhD from McMaster University, completed a Neurology residency at the University of Ottawa, and a Stroke Fellowship at the University of Calgary. He leads a clinical research program in acute stroke, intracerebral haemorrhage and early stroke recovery. The Ottawa Hospital, Civic Campus, 1053 Carling ave, Room C2182, Ottawa, ON K1Y4E9.

This work was supported by the University of Ottawa Brain and Mind Research Institute (D. D., D. C.), the Canadian Partnership in Stroke Recovery (D. D., D. C.), the University of Ottawa Department of Medicine (D. D.), a Canadian Institutes of Health Research Scholarship (M. P.), and an Ontario Graduate Scholarship (M. P.).

Submitted, 09 September 2016. Published as resubmitted by the authors on 03 October 2016. 